\title{
Type III Hyperlipoproteinemia Exaggerated by Sheehan's Syndrome With Advanced Systemic Atherosclerosis A 28-Year Clinical Course
}

\author{
Masa-aki Kawashiri, MD; Toshinori Higashikata, MD; Mutsuko Takata, MD; \\ Shoji Katsuda, MD; Kenji Miwa, MD; Atsushi Nohara, MD*; \\ Akihiro Inazu, MD**; Junji Kobayashi, MD ${ }^{\dagger}$; Masami Shimizu, MD; \\ Junji Koizumi, MD*; Hiroshi Mabuchi, MD
}

\begin{abstract}
A 38-year-old Japanese woman was admitted to hospital for further examination of systemic xanthomas. She had a past history of genital bleeding during her third delivery at the age of 21 years. She was diagnosed with Sheehan's syndrome. Her serum total cholesterol and triglyceride concentrations were 500 and $898 \mathrm{mg} / \mathrm{dl}$, respectively. She was also diagnosed as having type III hyperlipoproteinemia on the basis of the presence of a broad $\beta$-band on agarose gel electrophoresis and extremely high concentrations of very-low-density lipoprotein cholesterol $(310 \mathrm{mg} / \mathrm{dl})$. The diagnosis was later confirmed by her apolipoprotein E isoforms (E2/E2) and genotypes (epsilon2/epsilon2). Thyroid and corticosteroid hormone replacement therapy cured the xanthomas, but also elevated her blood pressure. The serum concentration of intermediate-density lipoprotein cholesterol was consistently high, whereas that of low-density lipoprotein cholesterol was relatively low during the follow-up. Coronary atherosclerosis had already developed by the age of 38 years, and progressed significantly over the following 28 years. Severe stenotic lesions were observed in the bilateral renal arteries and carotid arteries, and in the abdominal aorta when she was 66 years old. These findings suggest that the continuous elevation of intermediate-density lipoprotein cholesterol for a long period contributed to the development of the atherosclerotic lesions. (Circ J 2005; 69: 746-751)
\end{abstract}

Key Words: Atherosclerosis; Remnant lipoprotein; Sheehan's syndrome; Type III hyperlipoproteinemia

$\mathbf{E}$ pidemiological studies have established that high concentrations of total cholesterol and low-density lipoprotein (LDL) cholesterol, as well as triglycerides, are associated with increased risk of atherosclerosis! ${ }^{1-3}$ Type III hyperlipoproteinemia (HLP) is a rare inherited dyslipidemia characterized by the presence of $\beta$-migrating very-low-density lipoprotein remnants, ${ }^{4}$ and the concentrations of serum cholesterol and triglycerides are usually elevated to approximately $300 \mathrm{mg} / \mathrm{dl}$. The serum concentrations of LDL cholesterol, an atherogenic lipoprotein cholesterol, are usually lower than normal, whereas that of remnant lipoprotein cholesterol is markedly elevated. The common molecular cause of type III HLP is homozygosity for a rare isoform of apolipoprotein (apo) E, namely E2, whose single amino acid at residue 158 is changed from arginine to cysteines However, less than $10 \%$ of apoE2 homozygotes are known to develop hyperlipidemia, 6,7 which indicates that the manifestation of hyperlipidemia

(Received September 10, 2004; revised manuscript received December 8, 2004; accepted December 14, 2004)

Molecular Genetics of Cardiovascular Disorders, Division of Cardiovascular Disease, *Department of General Medicine, Kanazawa University Hospital, **School of Health Science, Faculty of Medicine and Department for Life-style-related Diseases, Graduate School of Medical Sciences, Kanazawa University, Kanazawa, Japan

Mailing address: Masa-aki Kawashiri, MD, Molecular Genetics of Cardiovascular Disorders, Division of Cardiovascular Disease, Graduate School of Medical Sciences, Kanazawa University, 13-1 Takara-machi, Kanazawa 920-8641, Japan. E-mail: masaaki@im2. m.kanazawa-u.ac.jp requires additional genetic, hormonal, or environmental factors, such as obesity, diabetes, hypothyroidism, or abnormal estrogen status? Patients with type III HLP have an increased risk for premature systemic and advanced atherosclerosis, despite having low concentrations of LDL cholesterol4, 8,9

To our knowledge, there are very few detailed reports on the long-term development and progression of atherosclerotic lesions in type III HLP. We report a woman with type III HLP (homozygous for apoE2) that was thought to be exaggerated by the onset of Sheehan's syndrome, and she developed advanced atherosclerotic lesions in the coronary, carotid and renal arteries, and abdominal aorta during a 28-year-clinical course.

\section{Case Report}

A 38-year-old Japanese woman was admitted to Kanazawa University Hospital in 1975 for examination of systemic xanthomatosis. At the age of 21 years in 1959 she had excessive bleeding during her third delivery, after which she become drowsy 7 days later and developed amenorrhea. She also lost her pubic and axillar hair, and became sensitive to cold. Her first cutaneous xanthoma was observed on her left thumb in 1971, after which they developed on her elbows, the backs and soles of her feet, and her knees. Xanthoma striatum palmare was also found (Fig 1A). Her serum total cholesterol and triglyceride concentrations were 500 and $898 \mathrm{mg} / \mathrm{dl}$, respectively, and lipoprotein analysis by agarose gel electrophoresis showed 

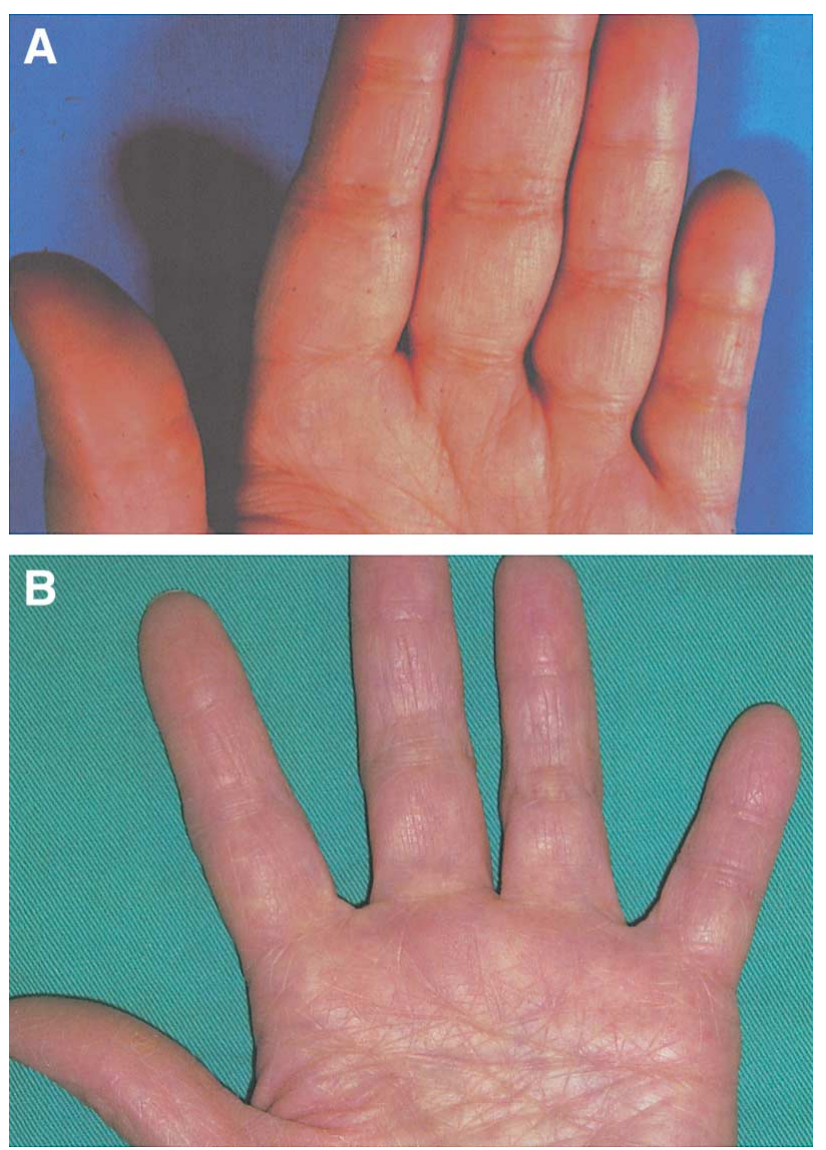

Fig 1. Cutaneous xanthomas on the fingers and palmar creases at the age of 38 years in 1975 (A), 17 years after the onset of Sheehan's syndrome. Striae palmaris was not evident in 2004 (B).

a broad $\beta$-band, suggesting the presence of type III HLP. The apoE phenotype of the patient was later determined to be apoE2/E2.

Several endocrine tests were performed to examine her anterior pituitary function (Fig 2) and the results showed that her anterior pituitary function, especially for thyroid stimulating hormone, was severely impaired, and because of the previous episode of genital bleeding she was diagnosed with Sheehan's syndrome.

She received $20 \mathrm{mg}$ of hydrocortisone and $70 \mathrm{mg}$ of thyroid sicca (Fig 3A). The serum total cholesterol and triglyceride concentrations were considerably decreased to approximately 200 and $150 \mathrm{mg} / \mathrm{dl}$, respectively, by dietary and hormone replacement therapy (HRT). Surprisingly, her palmar xanthoma began to disappear 2 months after starting HRT and had completely disappeared after 2 years of additional treatment with clofibrate (Fig 1B). However, the HRT increased her blood pressure above the normal range (Fig 3B). Lipoprotein analysis by agarose gel electrophoresis showed persistence of the broad $\beta$-band throughout the clinical course. In December 2003, the serum creatinine concentration increased gradually from approximately $0.6 \mathrm{mg} / \mathrm{dl}$ to $1.6 \mathrm{mg} / \mathrm{dl}$, and an ultrasonic examination revealed an atrophic left kidney. She was admitted to Kanazawa University Hospital for further examination of renal function and vascular complications in January 2004.

Her serum lipid concentrations at admission are shown
$\mathrm{GH}$

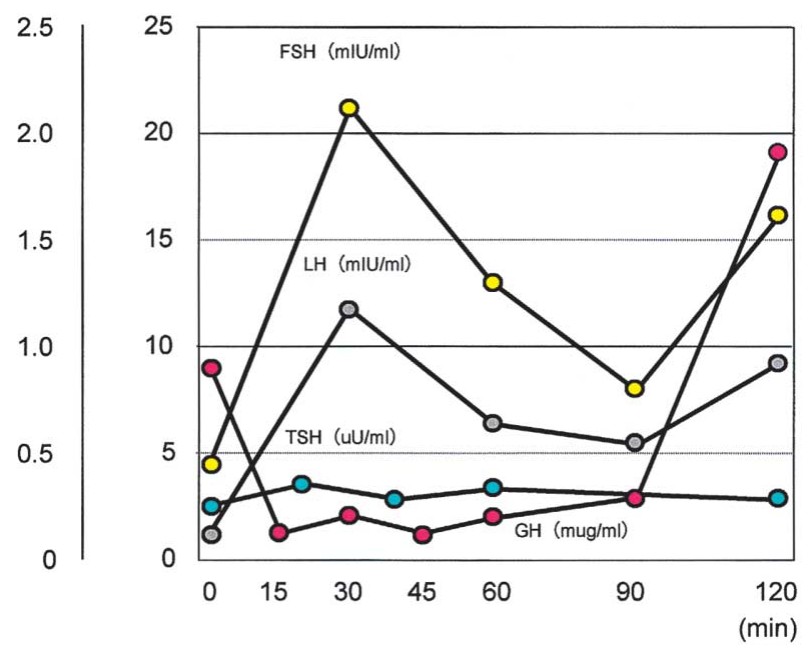

Fig 2. Results of thyroid hormone-releasing hormone test, luteinizing hormone-releasing hormone test, and arginine-loading test show no significant increases of thyroid stimulating hormone, luteinizing hormone, follicle-stimulating hormone, or growth hormone. The patient was diagnosed as Sheehan's syndrome on the basis of these results.

in Fig 4. Despite her serum cholesterol and triglyceride concentrations being within the normal limits, a significant amount of remnant lipoprotein was detected by electrophoresis and ultracentrifugation analysis. Coronary angiography performed in January 2004 showed significant progression of coronary atherosclerosis especially in the right coronary artery (Fig 5B), compared with the findings in 1976 (Fig 5A). Abdominal aortography and 3-dimensional computed tomography showed severe stenosis in the proximal portion of the left renal artery and occlusion of the right renal artery. A high degree of atheroma was present on the surface of the abdominal aorta (Fig 6). The intima-media thickness of the common carotid artery was $0.9 \mathrm{~mm}$, and atherosclerotic plaques were observed around the bilateral bifurcations of the carotid arteries. However, no significant intracranial atherosclerotic lesions were detected by magnetic resonance angiography (data not shown).

\section{Discussion}

The present case can be discussed from 2 viewpoints: (1) the etiological significance of anterior hypopituitarism to the onset of female type III HLP, and (2) higher concentrations of remnant lipoprotein independent of hyper-LDLcholesterolemia as a cause of systemic atherosclerosis.

The primary molecular cause of type III HLP is the presence of apoE24. The development of overt hyperlipidemia is known to require homozygosity of apoE2, although most apoE2 homozygotes present with normolipidemia or even hypolipidemia, 6,7 which indicates that the manifestation of type III HLP requires factors. This is the first case report of type III HLP caused by homozygosity of apoE2 exaggerated by Sheehan's syndrome, with secondary anterior hypopituitarism that resulted from the genital bleeding. In fact, type III HLP is much more common in males than in females, and atherosclerosis seldom develops in females until menopause.,10,11 There have been case reports of type III HLP 
A

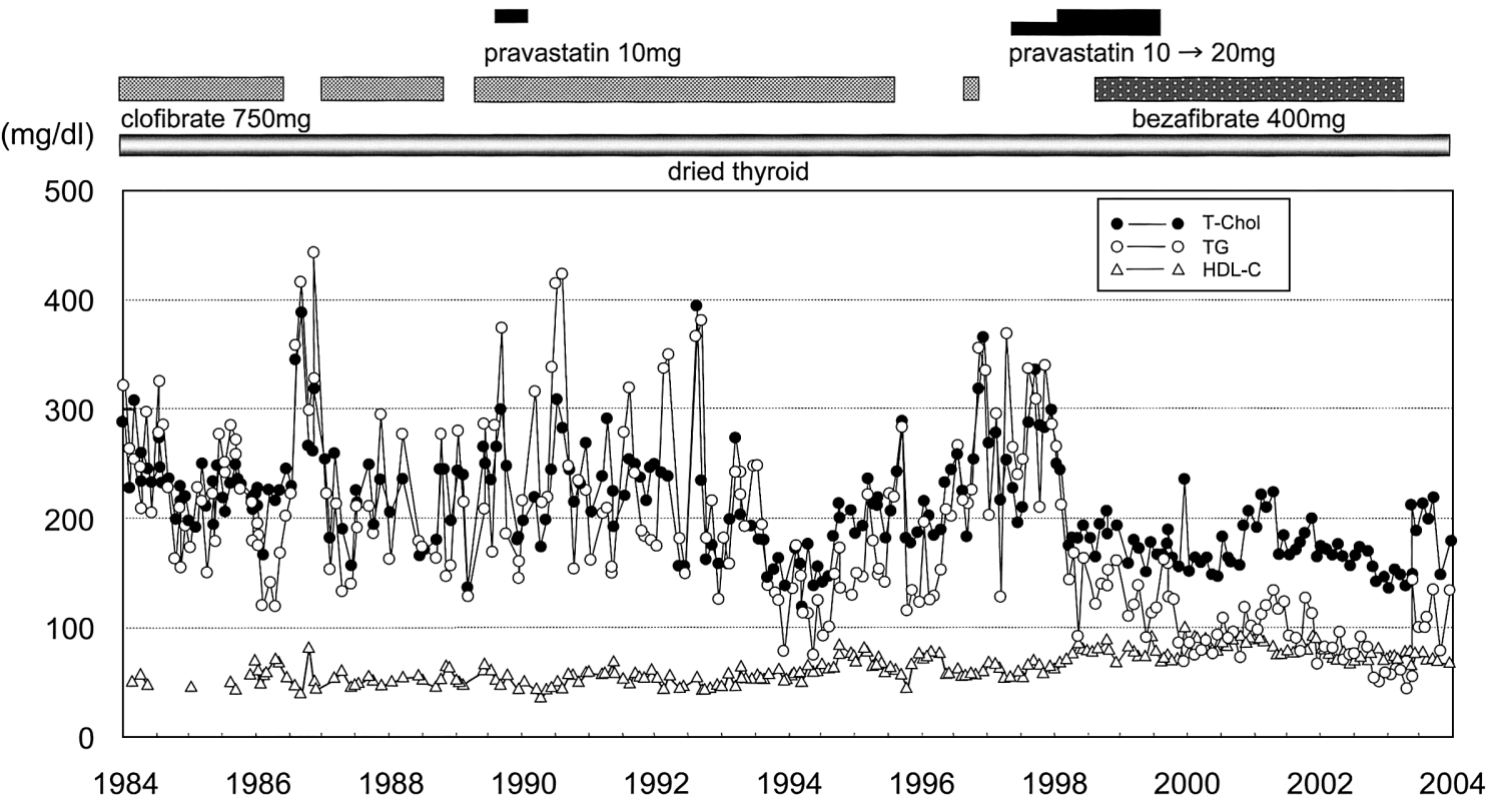

B
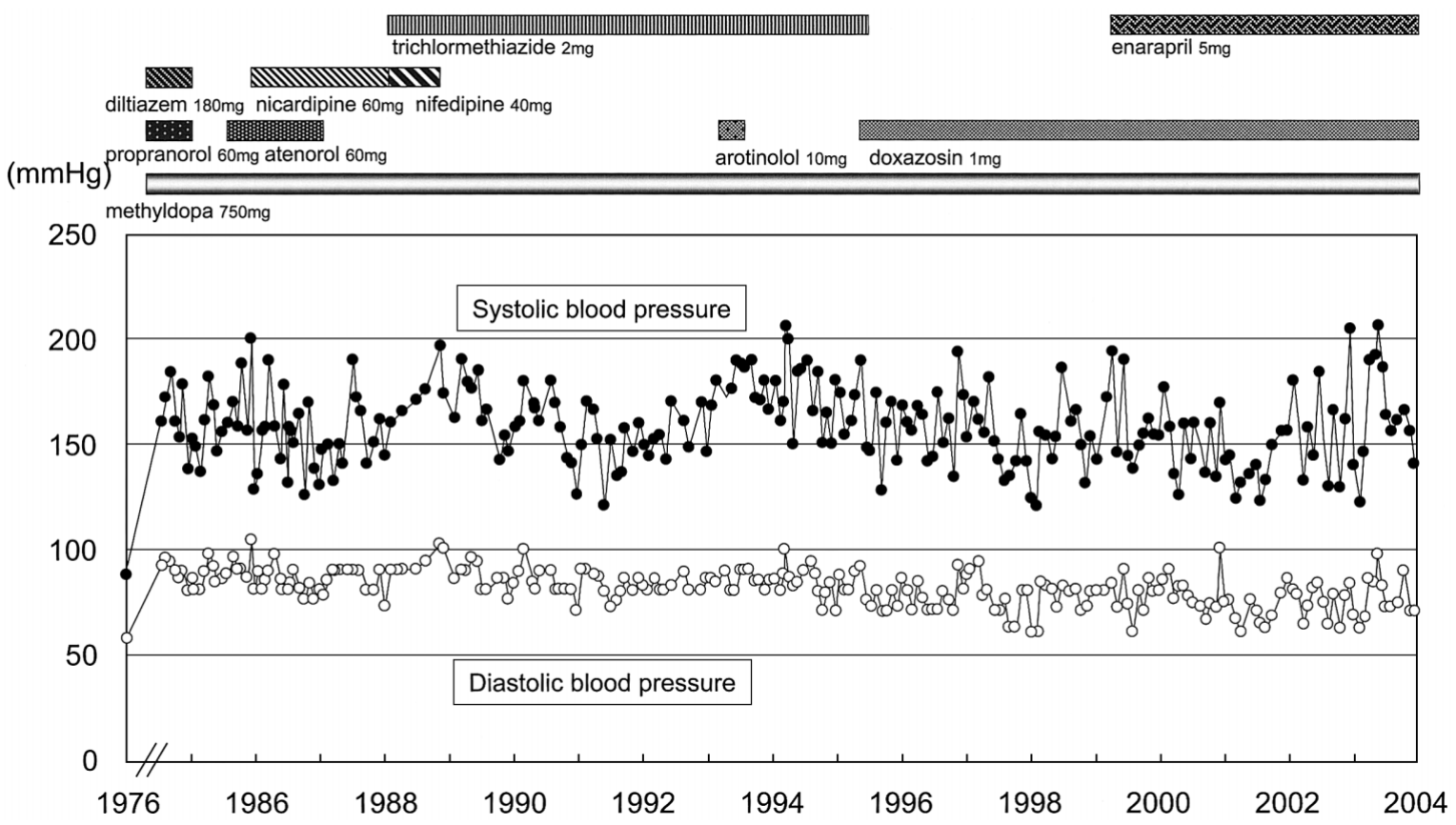

Fig 3. (A) Changes in serum concentrations of total cholesterol (T-Chol), triglycerides (TG), and high-density lipoprotein cholesterol (HDL-C). (B) Changes in blood pressure were treated with a combination of several hypotensive drugs. It was difficult to determine exactly when renovascular hypertension became clinically obvious because her blood pressure was constantly above the ideal range.

exaggerated by hypothyroidism in females, ${ }^{12-16} 3$ of which were associated with idiopathic early amenorrhea ${ }^{12-14}$ The present case also showed both hypothyroidism and pituitary amenorrhea. Nearly all the female patients with type III HLP in the previous reports were post-menopausal, with estrogen replacement returning the lipid profile to normal. Thus, estrogen insufficiency in addition to hypothyroid status might be important for the presentation of hyperlipidemia in apo E2/2 women.

It is very rare for premenopausal females to show clinically significant atherosclerosis even in familial hypercholesterolemia, ${ }^{17}$ a well known proatherogenic genetic disorder. Although most estrogen replacement therapy fails to ameliorate atherosclerotic cardiovascular diseases, endogenous estrogen is recognized as an atheroprotective hormone with the following effects on blood vessels: vasodilatation, inhibition of the response to vascular injury, increase in endothelial-cell growth, and smooth-musclecell growth ${ }^{18}$ Estrogen is also known to exert an atheroprotective effect on the lipid profile, by decreasing the total and LDL cholesterol concentrations and that of lipoprotein (a), and increasing the high-density-lipoprotein cholesterol and triglyceride concentrations 18 On the other hand, the role of testosterone in atherogenesis is controversial.19 In 


\begin{tabular}{|ll|r|}
\hline Parameters & $(\mathrm{mg} / \mathrm{dl})$ \\
\hline \hline Total cholesterol & 163 \\
Trigrycerides & 117 \\
HDL-cholesterol & 54 \\
LDL-cholesterol & 38 \\
RLP-cholesterol & & 9.1 \\
Apolipoprotein & A-I & 147 \\
& A-II & 25.4 \\
& B & 55 \\
& C-II & 5.3 \\
& C-III & 7.8 \\
& E & 8.3 \\
\hline \multicolumn{2}{|c|}{} \\
\hline \multicolumn{2}{|c|}{}
\end{tabular}

\begin{tabular}{|c|c|c|c|c|}
\hline & TC & FC & TG & PL \\
\hline Serum & 162 & 51 & 116 & 205 \\
\hline VLDL & 40 & 16 & 66 & 43 \\
\hline IDL & 27 & 10 & 18 & 22 \\
\hline LDL & 47 & 14 & 19 & 39 \\
\hline HDL & 49 & 13 & 17 & 104 \\
\hline
\end{tabular}
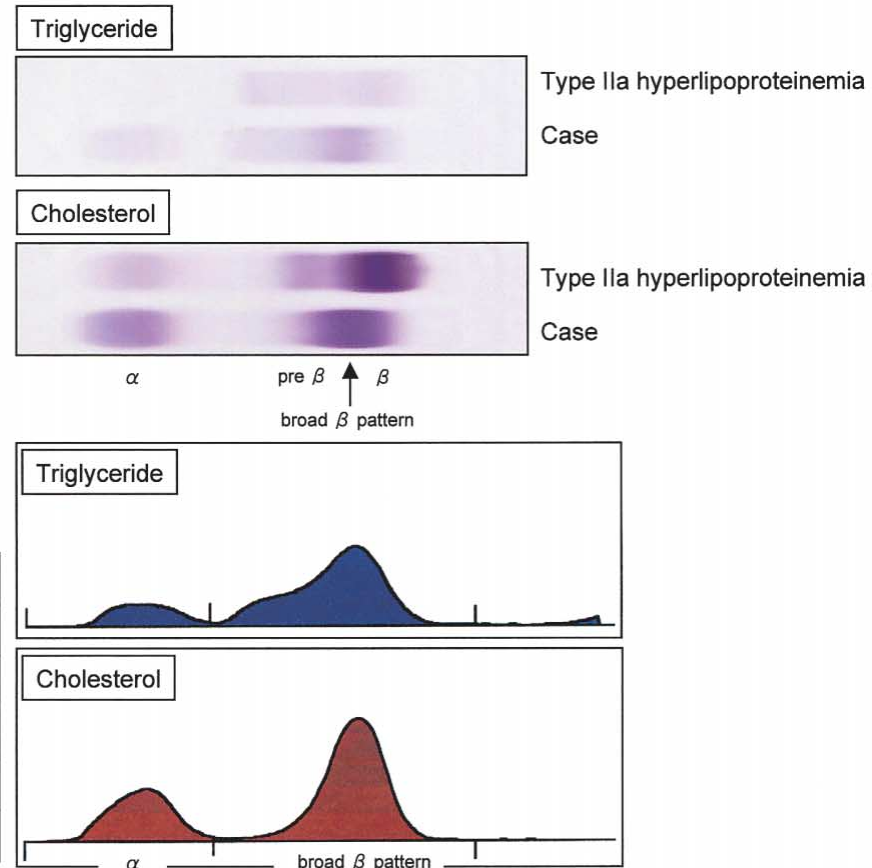

$(+)$

$(-)$

Fig 4. Lipoprotein analysis by agarose gel electrophoresis and ultracentrifugation in 2004. The patient had not taken lipid-lowering drugs for 3 months prior to the analysis, and her blood sample was drawn after overnight fasting. Serum concentrations of lipid and apolipoprotein (apo) concentrations were measured enzymatically. Low-density lipoprotein cholesterol (LDL-C) was measured by direct method using a commercially available kit (Daiichi), remnant-like lipoprotein-cholesterol (RLP-C) was measured after precipitating serum lipoproteins with anti-apoA-I and anti-apoB antibodies. Serum lipoprotein was separated by ultracentrifugation and agarose gel electrophoresis. Agarose gel electrophoresis showed a broad $\beta$-band. Serum concentrations of intermediate-density lipoprotein were continuously elevated despite her T-Chol and trigryceride concentration maintained within the normal ranges by dietary and drug treatment. VLDL, verylow-density lipoprotein; IDL, intermediate density lipoprotein; TC, total cholesterol; FC, free cholesterol; PL, phospholipids.

A

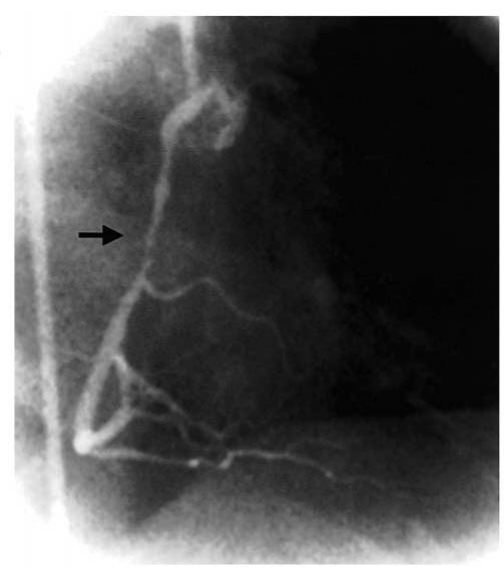

B

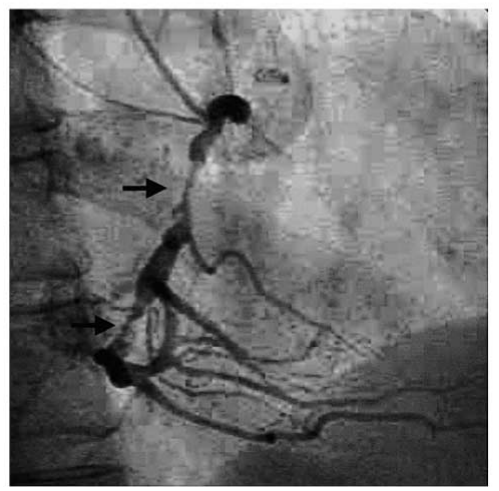

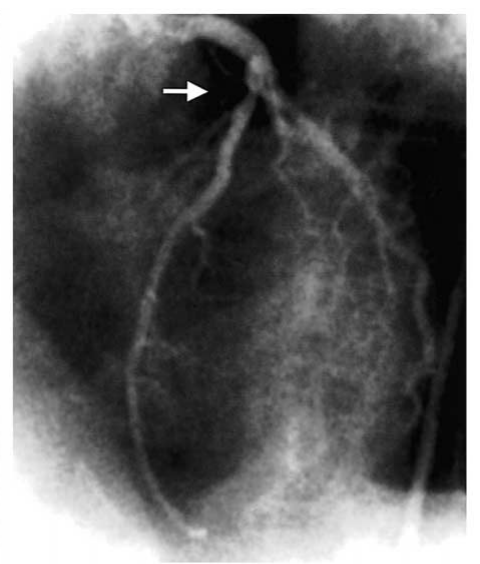

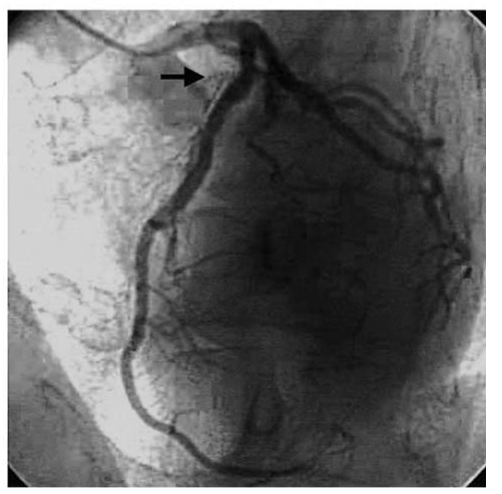

Fig 5. Coronary angiography in 1976 (A) and 2004 (B). In 1976, mild-to-moderate atherosclerotic lesions were observed in the proximal portion of the left anterior descending artery (approximate1y. $50 \%$ in diameter), the proximal portion of the posterolateral artery (approximately $25 \%$ in diameter), and the proximal-to-middle portion of the right coronary artery $(50-75 \%$ in diameter). In 2004, there was no significant angiographic progression of atherosclerotic lesions in the left coronary system, but $90 \%$ and $75 \%$ (diameter) stenoses had developed in the proximal and middle portions of the right coronary artery, respectively. 
A

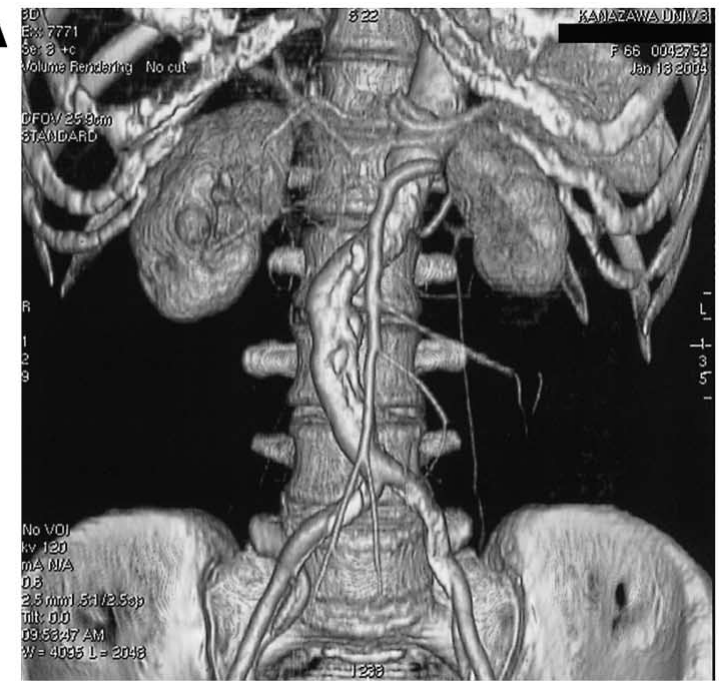

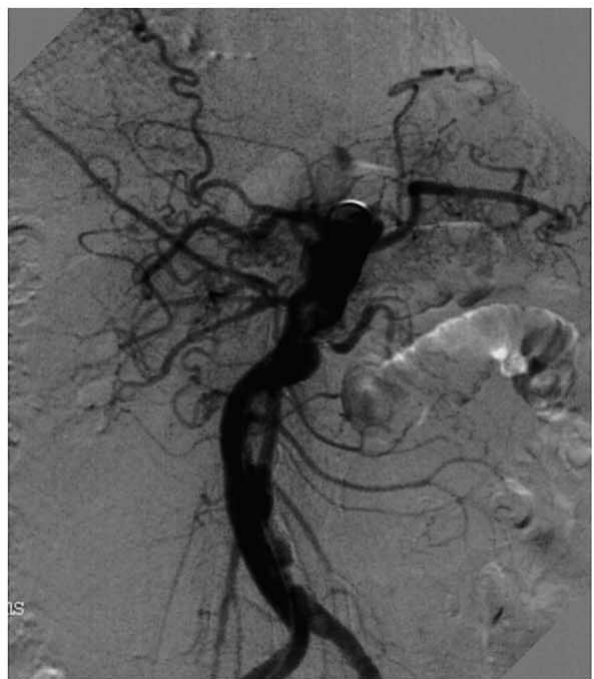

Fig 6. Atherosclerotic lesions in the abdominal aorta and bilateral renal arteries detected by 3-dimensional computed tomography (A) and angiography (B). The abdominal aorta has severe and complicated atherosclerotic lesions, the proximal and distal portions of the left renal artery are severely stenosed (approximately $90 \%$ in diameter) and there is total occlusion of the proximal portion of the right renal artery, which was compensated by collateral arteries from the lumbar arteries. The left kidney is atrophic and almost non-functional.

the case presented here, the concentrations of both estrogen and androgen were unphysiologically low (data not shown), and low sex hormone status in addition to type III HLP might play a role in the progression of systemic atherosclerosis.

The other important feature of the present patient was the development of premature coronary atherosclerosis and severe peripheral atherosclerosis, which provides strong evidence that factors other than LDL cholesterol concentration, such as remnant lipoproteins, contributed to the development and progression of atherosclerosis. It is difficult to presume when renovascular hypertension became clinically overt. Losito et al reported that renovascular hypertension increases in coronary artery disease more frequently than essential hypertension.20 Angiotensin II, which increased in a patient with renovascular hypertension, induced proatherogenic inflammatory cytokines such as interleukin- $6^{21}$ and antithrombotic factors such as plasminogen activator inhibitor- $1^{22}$ in vitro. It is therefore speculated that atherosclerotic renovascular hypertension rapidly accelerates the progression of systemic atherosclerosis.

Finally, premature coronary artery disease had already developed in the patient when she was only 38 years old, 17 years after the onset of Sheehan's syndrome, with apparent hyper-remnant-lipoproteinemia. Coronary and peripheral atherosclerosis progressed considerably despite the LDL cholesterol concentrations being kept within normal limits by dietary and clofibrate treatment. We presume that lipoproteins other than LDL, such as the remnant lipoproteins, that were continuously elevated during the clinical course, contributed to the development and progression of the marked atherosclerotic lesions in this patient. Anterior hypopituitarism and renovascular hypertension might also have accelerated systemic atherosclerosis.

\section{References}

1. Kannel WB, Castelli WP, Gordon T. Cholesterol in the prediction of atherosclerotic disease: New perspectives based on the Framingham study. Ann Intern Med 1979; 90: 85-91.
2. Martin MJ, Hulley SB, Browner WS, Kuller LH, Wentworth D. Serum cholesterol, blood pressure, and mortality: Implications from a cohort of 361,662 men. Lancet 1986; 2: 933-926.

3. Assmann G, Schulte H. Relation of high-density lipoprotein cholesterol and triglycerides to incidence of atherosclerotic coronary artery disease (the PROCAM experience): Prospective Cardiovascular Munster study. Am J Cardiol 1992; 70: 733-737.

4. Mahley RW, Rall SC Jr. Type III hyperlipoproteinemia (dysbetalipoproteinemia): The role of apolipoprotein $\mathrm{E}$ in normal and abnormal lipoprotein metabolilsm. In: Scriver CR, Beaudet AL, Sly WS, Valle D, editors. The metabolic \& molecular bases of inherited disease, $8^{\text {th }}$ edn. New York: McGraw-Hill; 2001; 2835-2862.

5. Schneider WJ, Kovanen PT, Brown MS, Goldstein JL, Utermann G, Weber W, et al. Familial dysbetalipoproteinemia: Abnormal binding of mutant apoprotein $\mathrm{E}$ to low density lipoprotein receptors of human fibroblasts and membranes from liver and adrenal of rats, rabbits, and cows. J Clin Invest 1981; 68: 1075-1085.

6. Rall SC Jr, Weisgraber KH, Innerarity TL, Mahley RW. Structural basis for receptor binding heterogeneity of apolipoprotein $\mathrm{E}$ from type III hyperlipoproteinemic subjects. Proc Natl Acad Sci USA 1982; 79: 4696-4700.

7. Davignon J, Gregg RE, Sing CF. Apolipoprotein E polymorphism and atherosclerosis. Arteriosclerosis 1988; 8: $1-21$.

8. Mahley RW, Innerarity TL, Rall SC Jr, Weisgraber KH. Lipoproteins of special significance in atherosclerosis: Insights provided by studies of type III hyperlipoproteinemia. Ann NY Acad Sci 1985; 454: 209-221.

9. Feussner G, Wagner A, Ziegler R. Relation of cardiovascular risk factors to atherosclerosis in type III hyperlipoproteinemia. Hum Genet 1993; 92: 122-126.

10. Morganroth J, Levy RI, Fredrickson DS. The biochemical, clinical, and genetic features of type III hyperlipoproteinemia. Ann Intern Med 1975; 82: 158-174.

11. Stone NJ. Secondary causes of hyperlipidemia. Med Clin North Am 1994; 78: $117-141$.

12. Krsek M, Ceska R, Horinek A, Horejsi B, Weiss V. Type III hyperlipoproteinaemia and primary amenorrhoea associated with severe hypothyroidism. Acta Paediatr 2000; 89: 1023-1024.

13. Kashyap AS, Anand KP. Primary amenorrhoea and xanthomatosis. Lancet 2004; 363: 616.

14. Mishkel MA, Nazir DJ, Crowther S. A longitudinal assessment of lipid ratios in the diagnosis of type III hyperlipoproteinaemia. Clin Chim Acta 1975; 58: 121-136.

15. Feussner G, Ziegler R. Expression of type III hyperlipoproteinaemia in a subject with secondary hypothyroidism bearing the apolipoprotein E2/2 phenotype. J Intern Med 1991; 230: 183-186.

16. Lindner MA, Illingworth DR. Expression of type III hyperlipoproteinemia in an adolescent patient with hypothyroidism. J Pediatr 1988; 113: 86-89. 
17. Mabuchi H, Koizumi J, Shimizu M, Takeda R. Development of coronary heart disease in familial hypercholesterolemia. Circulation 1989; 79: $225-232$.

18. Mendelsohn ME, Karas RH. The protective effects of estrogen on the cardiovascular system. $N$ Engl J Med 1999; 340: 1801-1811.

19. Wu FC, von Eckardstein A. Androgens and coronary artery disease. Endocr Rev 2003; 24: 183-217.

20. Losito A, Fagugli RM, Zampi I, Parente B, de Rango P, Giordano G, et al. Comparison of target organ damage in renovascular and essen- tial hypertension. Am J Hypertens 1996; 9: $1062-1067$.

21. Kranzhofer R, Schmidt J, Pfeiffer CA, Hagl S, Libby P, Kubler W. Angiotensin induces inflammatory activation of human vascular smooth muscle cells. Arterioscler Thromb Vasc Biol 1999; 19: $1623-1629$.

22. Kerins DM, Hao Q, Vaughan DE. Angiotensin induction of PAI-1 expression in endothelial cells is mediated by the hexapeptide angiotensin IV. J Clin Invest 1995; 96: 2515-2520. 